\title{
New Perspectives for Engineering Education - About the Potential of Mixed Reality for Learning and Teaching Processes
}

\section{Dr. Katharina Schuster, RWTH Aachen University}

Katharina Schuster has been working as a scientific researcher at IMA/ZLW \& IfU (IMA - Institute of Information Management in Mechanical Engineering, ZLW - Center for Learning and Knowledge Management \& IfU - Assoc. Institute for Management Cybernetics e.V.) of RWTH Aachen University since 2009.

She has completed her Master's Degree in Communication Sciences, Psychology and Political Sciences at RWTH Aachen University and has spent time abroad in the USA, Spain and Slovenia. Mrs. Schuster leads the research group Didactics in STEM Fields. Her main research interests are virtual learning environments, service-based learning and Open Innovation for University Management. In her dissertation (finished in 2014), she investigated the impact of immersive user interfaces on presence and learning.

\section{Prof. Anja Richert, RWTH Aachen University}

Prof. Dr. phil. Anja Richert

Managing Director of the Center for Learning and Knowledge Management (ZLW), RWTH Aachen University; Junior Professorship for Agile Management in Organization and Technology in the Faculty of Mechanical Engineering, RWTH Aachen University

Anja Richert took up the position of managing director of the Center for Learning and Knowledge Management of the RWTH Aachen University in February 2011. With a doctorate in Communication Science, she has been working at the IMA/ZLW \& IfU Institute Cluster since 2003, from 2008 to 2010 as manager of the Business and Research Division: Knowledge Management. In 2004 Anja Richert completed her degree in Communication Science with a distinction at the RWTH Aachen University. In December 2007, she gained her doctorate in the field of e-learning, likewise with a distinction. In the years 2010 and 2011 she received the International E-Learning Award (IELA) for the projects ROLE and RELOAD with the e-learning solutions developed under her leadership. Furthermore, she is a lecturer at the Mechanical Engineering Faculty of the RWTH Aachen University for a course on learning and work habits (compulsory elective subject for the degree course in Mechanical Engineering). Her main areas of research are the development of agile turbulence-tolerant processes and organizational concepts for knowledge and technology-intensive organizations, the development of knowledge management solutions and e-learning tools (e.g. knowledge maps) for various fields, as well as accompanying research for complex organizational development processes in heterogeneous research networks.

Prof. Sabina Jeschke, RWTH Aachen University 


\section{New Perspectives for Engineering Education - About the Potential of Mixed Reality for Learning and Teaching Processes}

In the field of engineering education, one important aspect lies on the cognitive processes of knowledge gain and knowledge transfer. In that context, the majority of mixed reality scenarios have been mainly the subject of game engines. 'Mixed Reality' describes the combination of virtual environments and natural user interfaces. Here, the user's field of view is controlled by his natural head movements via a head mounted display. Data gloves allow direct interaction with virtual objects and omnidirectional treadmills enable unrestricted navigation through a virtual environment by natural walking movements. Other tools as 3dimensional joysticks or sensor-enhanced clothes may come into play. To evaluate perspectives and potential for the use of mixed reality settings within engineering education an empirical study was carried out, focusing on the impact of spatial presence and flow on cognitive processes. Therefore an experimental research design was chosen. A mixed reality simulator (Virtual Theater) was used which combines two natural user interfaces: a head mounted display (HMD) and an omnidirectional treadmill. To assess the effects of natural user interfaces on cognitive processes, a two-group-plan (treatment and control group) was established. The mixed reality simulator was used as the main stimulus of the treatment group whereas the control group used a laptop to interact with the virtual environment. The learning environment was kept constant over both groups. The data were collected and interpreted with quantitative methods. Constraints of data collection consist as the influence of the hardware can only be evaluated within a set of independent variables, which consists of a combination of different user interfaces to a mixed reality simulator. Thereby not all of the disruptive factors could be eliminated. In this paper the study and the detailed results are described, which showed advantages especially regarding affective and motivational factors of virtual environments for cognitive processes. In particular, the depth of the resulting "spatial presence" and the phenomenon of "flow" are discussed. The paper closes with a discussion of the question, to what extend such innovative technologies establish new possibilities for educational sciences and pedagogics, especially focusing on engineering education and the field of virtual experiments.

\section{Introduction - New Perspectives for Engineering Education through Mixed Reality}

A main goal of engineering education is the development of professional skills, to support graduates with the application of their knowledge within their subsequent professional 
environment. A proper knowledge transfer is an important precondition for engineers to act competently and to solve different kinds of problems. However, due to the increasing number of study paths as well as the specialization of particularly technical oriented classes, there is a need for the integration of new media into the curriculum of most students [1]. Thus, the visualization of educational content in order to explain theory more concrete and tangible has gained importance. To prepare students adequately for new situations in their work life, virtual reality (VR) can be an effective instrument for learning and teaching processes. By imitating real-world processes, professional skills can be developed, increased or maintained. Especially if the learning process requires expensive equipment or usually would take place in a hazardous environment, the use of simulations is not only advantageous but necessary $[2,3]$. Apart from the software, the user interfaces of the technological systems applied in the simulation environment can affect the learning process as well [4]. One approach of improving learning with simulations is the development of natural user interfaces.

According to the classical memory theory, if the context in which knowledge is used i.e. in which it has to be transferred to new situations resembles the context in which the information has been learned in the first place, the memory works better. Moreover, how well we can retrieve knowledge from our long term memory depends on the quality of how well we encoded the information in the first place [5]. Sweller's cognitive load theory postulates that learning is as a task, which is partitioned in at least two parallel sub-tasks: Dealing with the content and controlling the learning environment with the respected user interfaces [6]. Therefore a lot of research and development activities follow the assumption that if the user can interface with the system in a natural way, more focus can be used for training than for the control itself [7].

However, to assume that hardware or software characteristics automatically lead to better learning outcomes is risky. Not every new approach which is technically feasible improves learning in the sense of task performance. The danger of designing complex and expensive virtual learning environments without having a positive impact on learning outcomes is obvious. However, judging the value of a virtual environment simply by its effect on task performance misses out on other factors which support learning. Boosting the students' motivation to deal longer, more steady or more effectively with the given content is also an important goal of virtual learning environments in engineering education [8,9]. Apart from learning outcome and motivation, a peak to a different domain reveals a third intended effect of virtual environments. According to the entertainment sector, the extent to which a game or 
in general a virtual environment can "draw you in" functions as a quality seal [2]. This phenomenon is often referred to as immersion [10].

Enabling natural movement as the most basic form of interaction is considered an important hardware quality to create immersion [11]. Manufacturers of hardware that are supposed to enhance immersion claim that "Moving naturally in virtual reality creates an unprecedented sense of immersion that cannot be experienced sitting down" [12]. Almost 20 years ago, this could already be confirmed by Slater [11]. Another basic assumption in the context of virtual learning environments and natural user interfaces is that greater immersion means better learning and potentially higher training transfer [4, 7]. This suggests that immersion would be the precondition for better learning, caused by the qualities of the user interfaces. However, if virtual environments are used in educational contexts, those assumptions need to be confirmed by empirical evidence. The presented study therefore focuses on the following questions:

- Do natural user interfaces create a higher sense of immersion?

- Do natural user interfaces lead to better learning?

- In what way to immersion and task performance interact in mixed reality learning environments?

If assessed in an experimental setting, the construct of immersion needs to be specified. Spatial presence and flow are considered key constructs to explain immersive experiences. In general, flow describes the involvement in an activity $[13,14]$, whilst spatial presence refers to the spatial sense in a mediated environment $[11,15]$. Spatial presence, as indicated in the name of the construct, refers to the spatial component of being immersed, i.e. the spatial relation of oneself to the surrounding environment. If we experience spatial presence in a mediated environment, we shift our primary reference frame from physical to virtual reality [15].

\section{Experimental analysis of the potential of Mixed Reality for Learning and Teaching Processes}

\subsection{Study Design - Focusing on spatial abilities}

The study presented in this paper assesses the relationship between objective hardware characteristics, subjective experiences and task performance. Their expected relationship is visualized in Figure 1. 


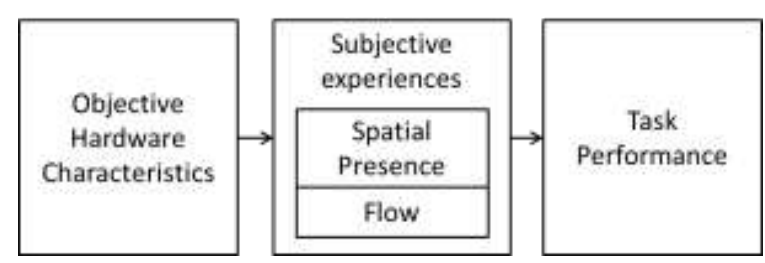

Fig. 1. Expected relationship between hardware characteristics, subjective experiences and task performance

All participants had to solve the same task in the same virtual environment, which was a large-scaled maze in a factory building. Within the maze, 11 different objects were located. The first task for the participants was to navigate through the maze and to imprint the positions of the objects to their memory. For that, they were given eight minutes of time. The second task was to recognize the objects seen before in the maze. The third task was to locate the positions of the objects on a map of the maze. This was done on a self-programmed application on a tablet (Nexus 10) with a drag-and-drop control mode. The view of the maze in the first and second task is pictured in Figure 2.

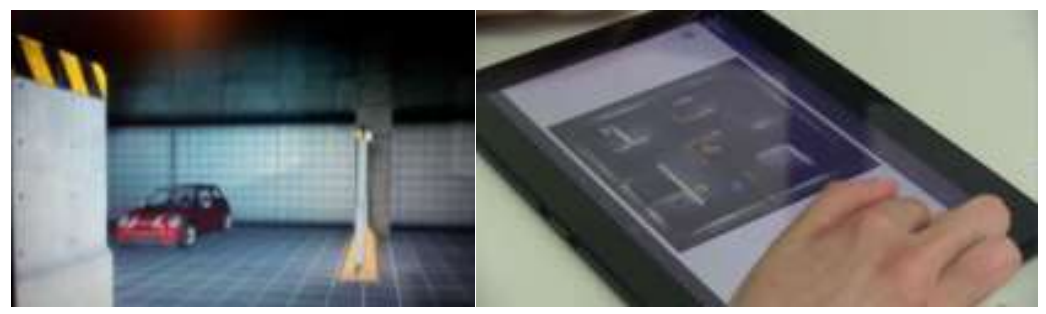

Fig. 2. View of the virtual environment used in the study in the first and in the third task

For both groups, the participants were given the chance to explore a test scenario (an italian piazza) freely for about three minutes before the actual task started. This was in order to get used to the respected control mode. All experimenters who conducted the experiments were trained in advance by experienced researchers. First they were being trained the functions of the hardware. In a second step, they took the observing position in a test run, and thirdly they conducted a test run on their own with the experienced researcher being the observer and giving feedback afterwards. Two groups of test persons were compared, having to use hardware which differed from each other regarding the following characteristics:

- control mode of the field of view,

- control mode of locomotion,

- display and

- body posture of the user. 
In the presented study, learning in a mixed reality simulator was compared to a somehow conventional learning with a laptop. The technical equipment is described in more detail in the following:

Laptop. The type being used was a Fujitsu Lifebook S761 with a 13,3 inch display and a 1366x768 display resolution. The field of view was controlled with a mouse. Locomotion was controlled by WASD-keys, where W/S keys controlled forward and backward while A/D keys controlled left and right. The hardware usually results in a sitting body posture while using the device.

Virtual Theatre. The Virtual Theatre is a mixed reality simulator which enables unrestricted movement through a virtual environment and therefore is used in an upright body posture. The user can move around within the environment by just walking in the desired direction. Therefore the control mode of locomotion is walking naturally. To track the movements of a user, the virtual theatre is equipped with 10 infrared cameras. They record the position of designated infrared markers attached to the HMD and an additional hand tracer. The components of the Virtual Theatre which came to use in the study are pictured in Figure 4. For a more detailed and complete description of the technical system see Ewert et al. [2] and Johansson $[7,18]$.

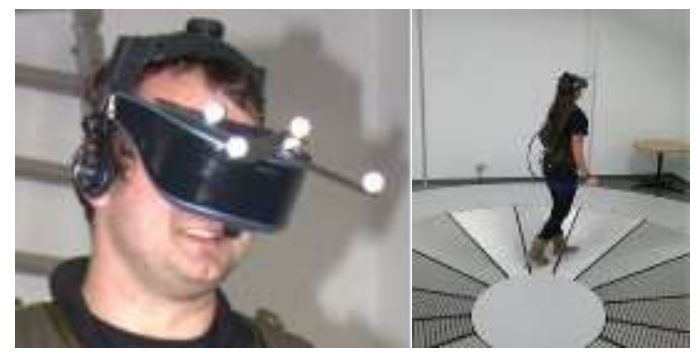

Fig. 4. Head Mounted Display and omnidirectional floor of the Virtual Theatre

Due to the composition of the simulator which was applied in the study, the hardware characteristics could only be tested in a certain combination and could not be isolated any further. The whole experimental procedure took one hour. The complete procedure is visualized in Figure 3. 
of the study was that natural user interfaces should have a positive effect on subjective experiences during the learning situation as well as on learning outcome, in our case operationalized in task performance.

The set of hardware characteristics functioned as the first independent variable in the presented study. Furthermore, interest in digital games (second independent variable) was measured before the first task. As dependent variables, spatial presence and flow were measured after the first task which had to be fulfilled either in the Virtual Theatre or at the laptop. As dependent measures of task performance, three different variables were analyzed: The number of objects that were correctly recognized in the second task, the third task reaction time and the accuracy of locating the objects on the map in the third task.

\subsection{Sample and Results}

A total of 38 students between 20 and 33 years $(M=24.71 ; S D=3.06 ; n=13$ female $)$ volunteered to take part in the study. The sample therefore represents a potential user group of virtual environments in higher education. They responded to a call for participation which was hung out at bulletin boards throughout the university but also posted on the front page of the virtual learning platform of the university and on several research and learning related blogs, social media platforms and news feeds. As an incentive and as a sign of appreciation, all participants took part in a drawing for a cordless screwdriver. All participants were healthy and highly interested in participating in the study. They did not report suffering from any physical or mental disorders. To rule out effects due to ametropia, participants were asked in advance to bring their corrective lenses just in case. If participants had been assigned to the Virtual Theatre group, they were asked to wear sturdy shoes.

Hypotheses regarding influences of hardware conditions on subjective experiences and task performance measures were tested with ANOVAs. With regard to the effects of the Virtual Theatre and the laptop on flow, significant differences were found $(F(1,36)=4.18 ; p<.05)$. Thus more flow has been experienced in the Virtual Theatre (see Fig. 6). Taking a closer look on subscales there is a highly significant difference between conditions in self-reported absorption $(\mathrm{F}(1,36)=10.63 ; \mathrm{p}<.01)$, but not in smooth and automatic running. There are also effects of hardware conditions on spatial presence. Self location in the Virtual Theatre was rated significantly higher $(\mathrm{F}(1,36)=15.79 ; \mathrm{p}<.001)$, which refers to the feeling of actually being in the virtual environment. Similarly, participants in the Virtual Theatre showed higher scores on the possible actions subscale of spatial presence $(F(1,36)=4.90 ; p$ $<.05$ ). There were no further significant effects regarding spatial presence (see Fig. 6). 

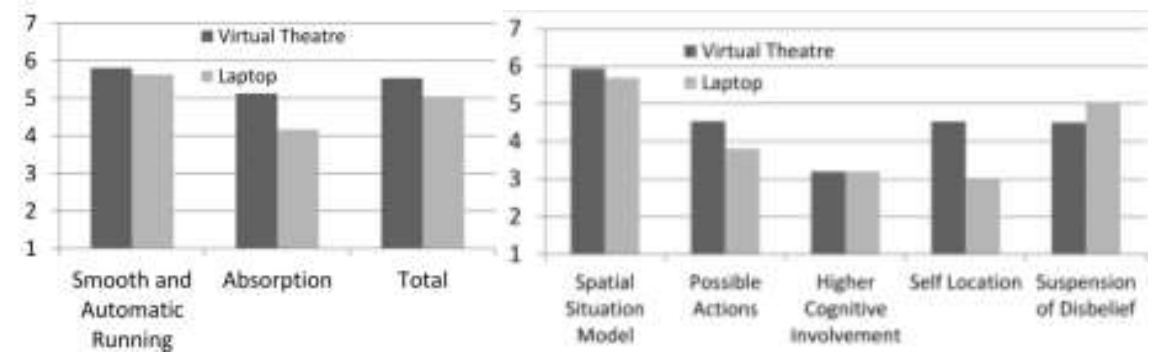

Fig. 6. Effects of Objective Hardware Characteristics on Flow and Spatial Presence

In addition to that we calculated the effects of hardware on task performance measures. In the recognition task of the objects from the virtual environment, participants in the Virtual Theatre condition made significantly more errors $(F(1,36)=10.93 ; p<.01)$, which opposes the hypothesis that the use of natural user interfaces leads to better learning outcomes (see Fig. 7). There were no differences regarding time on task and deviation between the two treatment conditions.

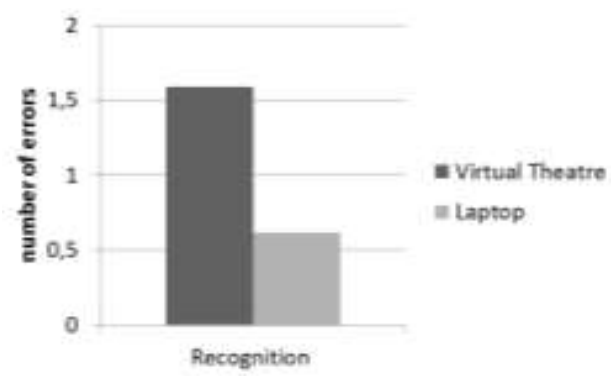

Fig. 7. Effects of Objective Hardware Characteristics on Task Performance, here: Recognition of previously presented objects

Regarding the question, in what way immersion and task performance interact in mixed reality learning environments, a significant interaction between flow and hardware characteristics was found in the case of the task performance indicators "deviation" $(\mathrm{F}(1,36)$ $=9.53 ; \mathrm{p}<.01$, see figure 8$)$ and "total duration" $(\mathrm{F}(11,36)=4.65 ; \mathrm{p}<.05$, see figure 8$)$. In other words, high values of experienced flow subside with better task performance, if a laptop has been used for learning, but with worse task performance if a mixed reality simulator has been used. 

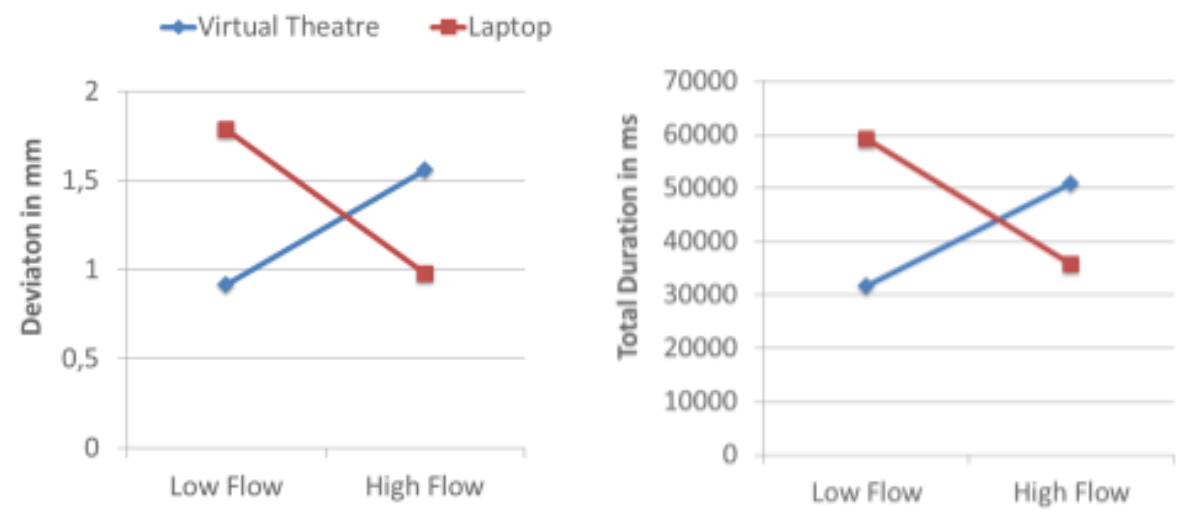

Fig. 8: Interaction between flow and user interface according to deviation and total duration (shown in percentile split)

\section{Discussion}

Concerning the effects of natural user interfaces, the results show that mixed reality learning environments indeed lead to more flow. This is due to a higher self-reported level of absorption in the experimental group. Although flow is an activity related construct, this result is in line with the theoretical assumptions that hardware which allows natural walking can support the feeling of "diving" into the virtual environment, which in general terms is often referred to as immersion.

Next, the effects of the hardware on spatial presence are analyzed in more detail. Students who used the Virtual Theatre reported a higher self-location in the virtual environment which indicates that they had shifted their primary reference frame from the physical to the virtual world. Although the given task didn't require any further non-mental actions but navigating through the virtual environment, students in the Virtual Theatre reported higher on the subscale of possible actions. However, for the other subscales, no differences were measured.

The interaction of flow and hardware characteristics in the case of the task performance indicators "deviation" and "total duration" leads to the assumption that in a mixed reality simulator, the subtasks of learning "switch roles": Controlling the mixed reality environment including the phenomenon of "diving in" becomes the main task, while dealing with the actual content-related task moves gets less attention [6]. Although the given data set gives no insight to the question, if this is a conscious or a subconscious decision, this is a result of high importance in engineering education. Especially if the trend of developing immersive learning environments continues, the possibility for students to get used to the control mode is absolutely necessary in order not to inhibit the learning process. 
According to the results of this study, immersion is not the precondition for better learning in virtual environments with natural user interfaces. Thus, the underlying model of the study (see Fig. 1) needs to be adjusted for further research. The only effect of the Virtual Theatre on task performance was a negative influence on recognition. This result is contradictory to the assumption that immersion leads to better learning. It seems that controlling the hardware was less intuitive than expected. This probably lead to the typical situation for learning with virtual environments: Dividing the available cognitive resources on the two parallel sub-tasks of dealing with the content and controlling the learning environment with the respected user interfaces [6]. Moreover, the combination of an HMD and real physical locomotion could lead to cognitive dissonance. When wearing the HMD, the user can see where he or she walks within virtuality, but not within physical reality. Therefore the user takes a risk and has to trust in the technology in order to continue his or her actions. Last but not least, walking on the omnidirectional floor is a new experience for users and therefore could result in the fear of falling. All interpretations for the given results are going to be addressed in a follow-up study, where previous participants of the study will be interviewed on their experiences.

Finally, some limitations of the present study are considered that should be pursued for future research. One limitation refers to the type of hardware examined. Since the different technical characteristics of the Virtual Theatre can only be tested in a set, it is not possible to isolate single effects. The other aspect concerns the task chosen for this experiment. Low levels of cognitive involvement in both groups indicate that the whole sample might not have been challenged enough. Since challenge is an important precondition for the motivation for learning, more challenging tasks are going to be tested in the future.

This exploratory study on the effects of mixed reality learning environments on subjective experiences and task performance confirmed a few theoretical assumptions but also contradicted others. In a next step, interviews with participants from both groups are going to be conducted. A deeper insight on the participants' experiences will allow a more differentiated view on the subject of our research.

Acknowledgements. The present work is supported by the Federal Ministry of Education and Research within the project "Excellent teaching and learning in engineering sciences (ELLI)". The authors would like to thank Prof. Martina Ziefle for constant advice on the presented work. 


\section{References}

[1] Kerres, M.: Mediendidaktik. Konzeption und Entwicklung mediengestützter Lernangebote. Munich 2012.

[2] Ewert, D., Schuster, K., Schilberg, D., Jeschke, S.: Intensifying learner's experience by incorporating the virtual theatre into engineering education,. In: Proceedings of the IEEE Educon Conference, Berlin: 13. - 15. March 2012.

[3] Malkawi, S., Al-Ariadah, O.: Students` assessment of interactive distance experimentation in nuclear reactor physics laboratory education. In: European Journal of Engineering Education, Vol. 38, No. 5, pp. 512 - 518. (2013).

[4] Witmer, B.G.; und Singer, M.J.: Measuring Presence in Virtual Environments: A Presence Questionnaire, In: Presence: Teleoperators and Virtual Environments, Vol. 7, Nr. 3, pp. 225 - 240. (1998)

[5] Zimbardo, P.G., Gerrig, R. J.: Psychologie, 7th Edition. Berlin, Heidelberg, New York: Springer (2003)

[6] Sweller, J.: Element Interactivity and Intrinsic, Extranous, and Germane Cognitive Load. Educational Psychology Review, 22 (2), S. 123 - 138 (2010)

[7] Johansson, D.: Convergence in Mixed Reality-Virtuality Environments - Facilitating Natural User Behaviour. Örebro Studies in Technology 53 (2012)

[8] Hebbel-Seeger, A. (2012): Motiv: Motivation?! - Warum Lernen in virtuellen Welten (trotzdem) funktioniert. In: Lernen und Lehren in virtuellen Welten. Zeitschrift für E-Learning - Lernkultur und Bildungstechnologie, Vol. 1/2012, pp. 23 - 35. Innsbruck: Studienverlag.

[9] Müller, F.H. (2006): Interesse und Lernen. Report - Zeitschrift für Weiterbildungsforschung 29, 1, pp. 48 62

[10] Murray, J.: Hamlet on the Holodeck: The Future of Narrative in Cyberspace (1997)

[11] Slater, M.: Taking Steps: The Influence of a Walking Technique on Presence in Virtual Reality. In: ACM Transactions on Computer-Human Interaction, Vol. 2, No. 3, September 1995, pp. 201-219.

[12] Virtuix Technologies, Online available at: http://www.virtuix.com/

[13] Csikszentmihalyi, M., LeFevre, J.: Optimal experience in work and leisure. In: Journal of Personality and Social Psychology, 56, pp- 815 - 822 (1989)

[14] Rheinberg, F.; Engeser, S.; Vollmeyer, R.: Measuring components of flow: the Flow-Short-Scale. In: Proceedings of the 1st International Positive Psychology Summit, Washington DC, USA (2002)

[15] Slater, M.: Measuring Presence: A Response to the Witmer and Singer Presence Questionnaire, In: Presence: Teleoperators and Virtual Environments, Vol. 8, Nr. 5, pp. 560 - 565 (1999)

[16] Vorderer, P, Wirth, W., Gouveia, F. R., Biocca, F., Saari, T., Jäncke, F., Böcking, S., Schramm, H., Gysbers, A., Hartmann, T., Klimmt, C., Laarni, J., Ravaja, N., Sacau, A., Baumgartner, T. \& Jäncke, P.: MEC Spatial Presence Questionnaire (MEC-SPQ): Short Documentation and Instructions for Application. Report to the European Community, Project Presence: MEC (IST-2001-37661). Online. Available from http://www.ijk.hmt-hannover.de/presence. (2004)

[17] Edelmann, W. (1996): Lernpsychologie (5th Edition). Weinheim: Beltz PVU

[18] Johansson, D., de Vin, L.J.: Towards Convergence in a Virtual Environment: Omidirectional Movement, Physical Feedback, Social Interaction and Vision. In: Mechatronic Systems Journal, November Issue (2011) 